\title{
Urbs oblivionalis Urban Spaces and Terrorism in Italy
}

di Elena Pirazzoli e Roberto Zancan

\begin{abstract}
The 14th International Architecture Exhibition (2014), titled Fundamentals and directed by Rem Koolhaas, presented a section called Monditalia: 41 architectural projects concerning specific case studies but together forming a comprehensive portrait of Italy.

Urbs oblivionalis. Urban Spaces and Terrorism in Italy was the research focusing the planning reaction after terroristic attacks occurred in this country between 1961 and 1993. In this text the two authors underline the essential points of their research, still open and sadly actual.
\end{abstract}

\section{Keywords}

Architecture, Terrorism, Urban Studies, Design, Italy 


\section{Ocula ${ }^{\text {Plux__Architettura }}$}

Elena Pirazzoli e Roberto Zancan | Urbs Obvionalis

Questa ricerca, effettuata per essere esposta nella $14^{\mathrm{a}}$ Biennale di Architettura di Venezia, tenta di ridurre nello spazio di un'installazione un tema "irriducibile". Irriducibili sono stati definiti alcuni dei protagonisti della stagione del terrorismo italiano, irremovibili nelle loro posizioni ideologiche. Irriducibile, nel senso di impossibile da contenere, è la doppia stagione, sovrapposta ma allo stesso tempo essenzialmente differente, degli "anni di piombo" e della "strategia delle tensione". E irriducibile, nel senso di frattura non componibile, è il suo portato personale, familiare, storico, sociale e secondo il peculiare punto di vista di questa ricerca - urbano.

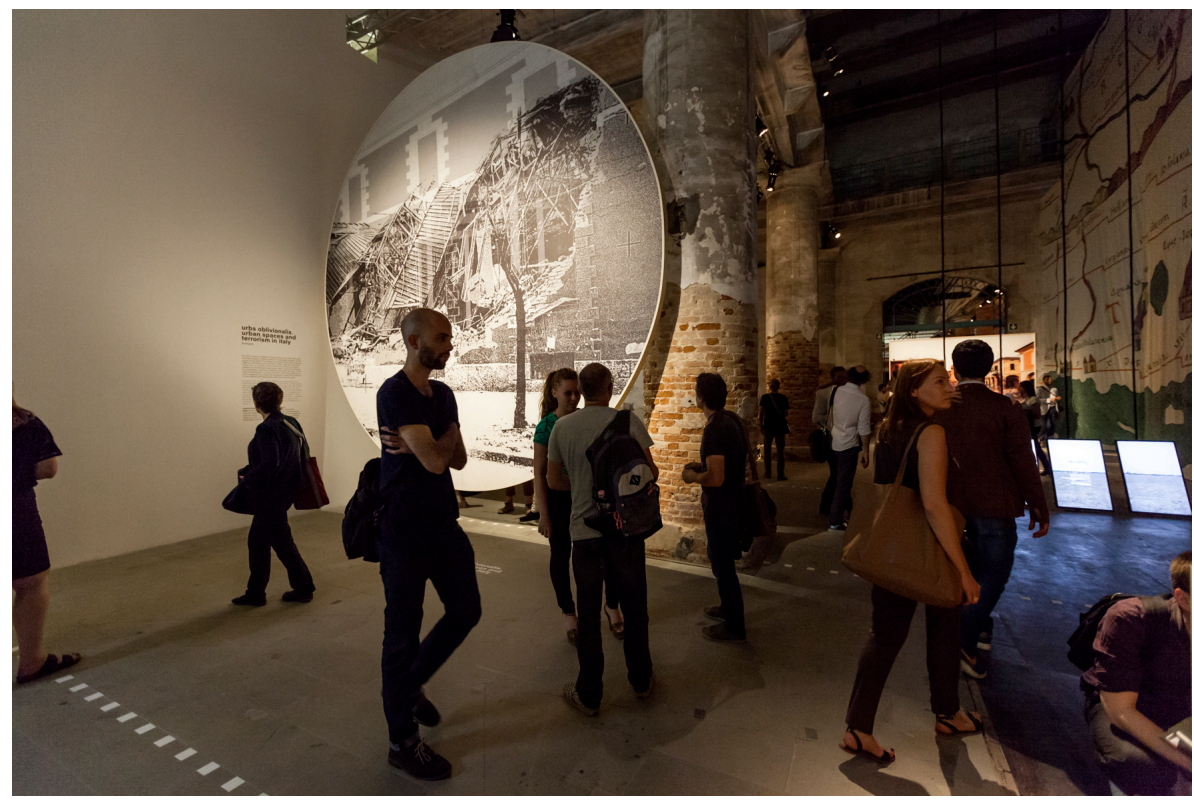

Approcciando per la prima volta il complesso tema della violenza politica in Italia nell'ampio arco di tempo che va dall'inizio degli anni Sessanta fino all'inizio degli anni Novanta sono i dati a colpire: secondo il Ministero dell'Interno tra il 1969 e il 1987 ci furono 14.591 atti di violenza "politicamente motivati". Atti spesso "minori", ma indicativi di un clima sociale diffuso, di cui le bombe di Milano, Brescia, Bologna furono gli episodi maggiori.

Tuttavia, l'obiettivo di questa ricerca non è la ricostruzione storica né dei fatti, né del clima politico. L'approccio a quella stagione è differente: il coinvolgimento, la ripercussione di questi atti violenti politicamente motivati sulla dimensione urbana e sulle discipline del progetto.

L'architettura viene chiamata in causa ovviamente "dopo" una strage o un attentato. Per ripristini funzionali, restauri o progettazione di forme/spazi memoriali. Non sempre questi momenti sono tra loro in relazione o tutti presenti: in particolare, è il tema della progettazione di luoghi e segni del ricordo a essere affidata molto spesso a segni minimi (come lapidi) o addirittura spontanei. I memoriali progettati sono pochissimi e, quasi sempre, fortemente improntati dalla lettura delle tracce lasciate dagli attentati, riprendendo la dimensione indiziaria e peritale. 


\section{Oculala ${ }^{\text {Plux__rchitettura }}$}

Elena Pirazzoli e Roberto Zancan | Urbs Obvionalis

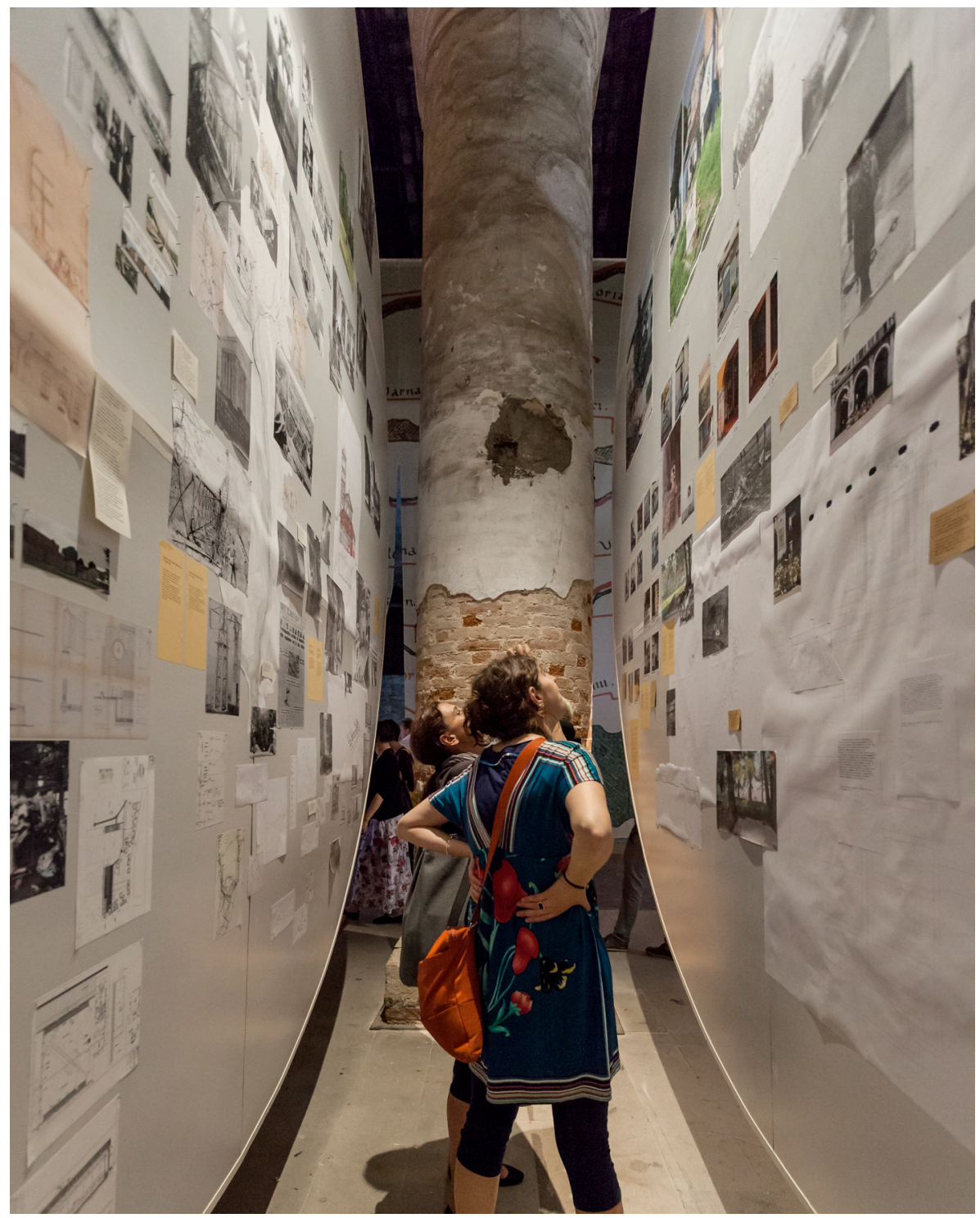

L'architettura, tuttavia, viene coinvolta anche nella definizione degli obiettivi degli attentati e delle stragi, che colpiscono luoghi di aggregazione, piazze, patrimonio artistico, infrastrutture.

Se cerchiamo di leggere questi fatti prescindendo dalla loro intrinseca e differente natura politica, ci accorgiamo, ad esempio, che quando si è voluto colpire l'Italia in modo radicale, lo si è fatto colpendo in sincrono Milano e Roma. Questo avvenne il 12 dicembre del 1969 a Piazza Fontana a Milano, ma anche all'Altare della Patria a Roma, anche se questo episodio si è perduto nella consapevolezza e nella memoria dell'opinione pubblica. Lo stesso accadde nella notte del 27 luglio 1993, con l'attacco al PAC in via Palestro a Milano e alle due chiese di San Giovanni in Laterano e San Giorgio al Velabro a Roma. Oppure, si è colpito ciò che congiunge Roma e Milano, le due "capitali" d'Italia, ovvero la linea ferroviaria Direttissima, nei suoi punti più 
cruciali: l'attraversamento appenninico presso la Grande Galleria che sbuca a San Benedetto Val di Sambro (nel 1974 con la bomba sul treno Italicus e nel 1984 con la bomba sul Rapido 904) e la stazione di Bologna.

Altre vicende sono invece meno note, trattate a livello locale o dimenticate. Alcune hanno avuto nel passato un forte peso sociale, hanno comportato spostamenti in massa dell'esercito o delle forze dell'ordine, coinvolgendo il territorio in modo totalizzante. È il caso dell'Alto Adige, con le bombe contro i tralicci dell'alta tensione durante la Feuernacht (11 giugno 1961) organizzata dal Befreiungsausschuss Südtirol ("comitato di liberazione del Südtirol") da un lato dell'Italia e della rivolta urbana di Reggio Calabria dall'altro (1970-1971).

Il display che abbiamo considerato più adeguato per rendere questa complessità presenta esternamente il tema in modo simbolico: sulle facce dei due grandi dischi appesi, rivolte al resto dell'esposizione, appare come iniziale impatto l'elaborazione di una fotografia del PAC scattata dalla Polizia scientifica di Milano con la sovrapposizione dell'edificio prima e dopo l'attentato; sul secondo disco, in bianco su fondo nero, risalta una citazione tratta da una testimonianza di Giovanni Brusca, che riporta una "battuta" del boss Antonino Gioè: "Che ne direste se un giorno vi svegliaste e non trovereste più la Torre di Pisa?". Una domanda riportata fedelmente, anche con l'errore di tempo verbale, per dare l'idea dell'ultima fase presa in considerazione dalla ricerca, durante la quale il patrimonio artistico del Paese venne coinvolto negli attacchi di Cosa Nostra allo Stato all'interno della presunta "trattativa Stato-mafia", su cui oggi si indaga.

Nelle facce interne dei grandi dischi sono esposti invece i materiali di una ricerca in divenire: l'estratto di una raccolta di documenti, fotografie, disegni, elaborazioni, che sono parte di un work in progress. La raccolta infatti è fortemente incompleta, dato che in questi mesi di lavoro abbiamo compreso quanto le riflessioni urbanistiche e architettoniche dei conflitti politici siano troppo complesse e contraddittorie per produrre un'interpretazione unica, chiara, incontrovertibile.

Questa ricerca, frammentata e aperta, può essere presentata solo nella forma di un nugolo di vicende multiple e parallele: strategie di attacco territoriale, impatto urbano e sociale di bombe e uccisioni, esempi di buoni e cattivi memoriali, condizioni dei luoghi. Ogni vicenda è narrata attraverso la stessa serie di documenti: un'archeologia di immagini iconiche dell'evento (in bianco e nero), disegni e diagrammi che analizzano e mostrano le relazioni tra l'attacco e l'uso dello spazio pubblico, una descrizione fotografica della presente situazione dei luoghi, copie di documenti d'archivio che testimoniano i restauri e/o i progetti memoriali.

Ritorni, connessioni, sovrapposizioni, ripetizioni, permettono di capire con quali questioni gli architetti devono confrontarsi quando si trovano a lavorare su luoghi colpiti da attacchi terroristici: quei fundamentals che devono usare per far vivere la città dopo i drammi.

Gli eventi violenti accadono ovunque. Ogni giorno dei luoghi sono attaccati per la loro qualità di spazi pubblici. Noi vogliamo che essi continuino ad essere tali, senza trasformarsi in monumenti. Questa ricerca sulla 


\section{Ocula ${ }^{\text {Plux__Architettura }}$}

Elena Pirazzoli e Roberto Zancan | Urbs Obvionalis

Re(s)pubblica Italiana assume significato solo se diviene capace di ispirare ricerche simili anche in altri Paesi, per mostrare quanto abbiamo bisogno di un approccio antiretorico rispetto agli eventi politici drammatici. Alcune delle questioni che suggeriamo ai progettisti del futuro sono: come rendere comprensibile quello che è accaduto? Come aiutare ad assorbirlo/elaborarlo consapevolmente e dimenticarlo - più che ricordarlo - per dare una possibilità di continuazione della vita? Come lasciare un segno e continuare a usare questi luoghi come spazi collettivi? Più che un'arte della memoria, servirebbe per le nostre città quella che Umberto Eco ha definito un'ars oblivionalis. Un'arte che, anziché congelare i luoghi nel ricordo, "dovrebbe porre i principi non per ovviare a, ma per produrre il deterioramento della traccia attraverso un progetto volontario". Dimenticare per fare affiorare il ricordo. Ciò è tanto più vero per la "stagione del terrorismo", dove luoghi, edifici e muri diventavano obiettivi proprio in virtù delle loro qualità di luoghi comuni, quotidiani, banali.

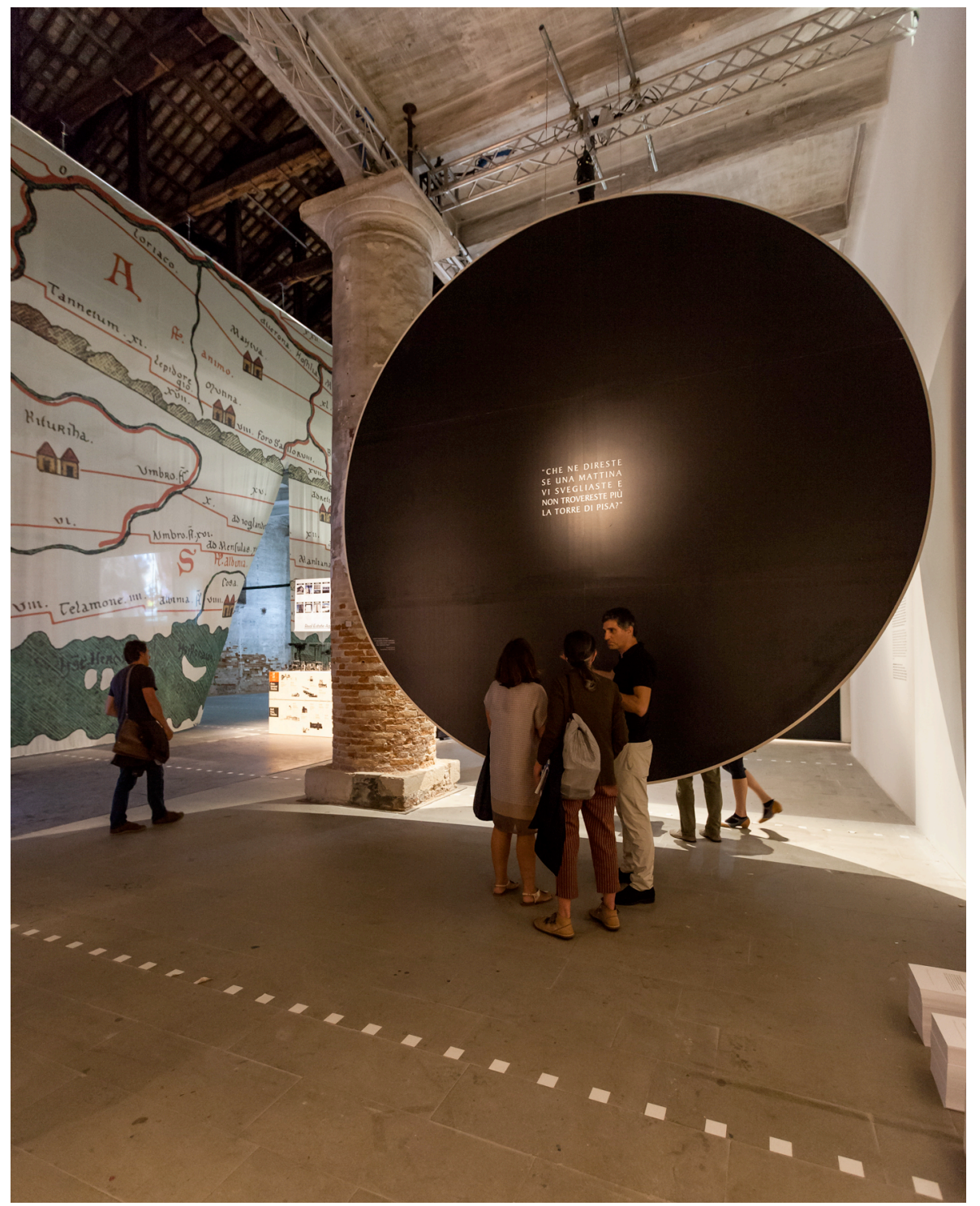

5 | www.ocula.it | Marzo 2016 